\title{
The Coulomb Phase Shift Revisited
}

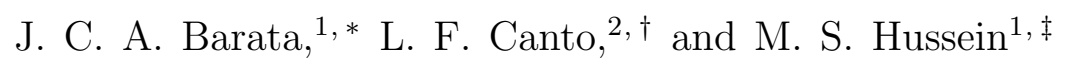 \\ ${ }^{1}$ Instituto de Física, Universidade de São Paulo, \\ C.P. 66318, 05314-970 São Paulo, SP, Brazil \\ ${ }^{2}$ Instituto de Física, Universidade Federal do Rio de Janeiro, \\ C.P. 68528, 21941-972 Rio de Janeiro, RJ, Brazil \\ and \\ Centro Brasileiro de Pesquisas Físicas (CBPF), \\ Rua Xavier Sigaud, 150, 22290-180 Rio de Janeiro, RJ, Brazil
}

\begin{abstract}
We investigate the Coulomb phase shift, and derive and analyze new and more precise analytical formulae. We consider next to leading order terms to the Stirling approximation, and show that they are important at small values of the angular momentum $l$ and other regimes. We employ the uniform approximation. The use of our expressions in low energy scattering of charged particles is discussed and some comparisons are made with other approximation methods.
\end{abstract}

PACS numbers: 25.60.Pj, 25.60.Gc

Keywords: Coulomb scattering, phase shifts, semiclassical approximation

\section{INTRODUCTION}

The customary procedure to deal with charged particle scattering is to partial wave the amplitude and identify the Coulomb phase shift from which scattering information can be obtained, by adding to the Coulomb amplitude, the contribution from whatever other shortrange potential. In many applications, the asymptotic form of large angular momentum is employed for the Coulomb phase shift. In this paper we revisit the derivation of this asymptotic and derive next to leading order correction to the usual WKB from.

\footnotetext{
*Electronic address: jbarata@if.usp.br

${ }^{\dagger}$ Electronic address: canto@if.ufrj.br

${ }^{\ddagger}$ Electronic address: hussein@if.usp.br
} 
First, we recall the form of the Coulomb phase shift [1]

$$
e^{2 i \sigma_{l}}=\frac{\Gamma(1+l+i \eta)}{\Gamma(1+l-i \eta)}
$$

where $\Gamma$ is Euler's gamma function, $l$, a non-negative integer, is the angular momentum and the real parameter $\eta$ is the so-called Sommerfeld parameter, which is inversely proportional to the square root of the scattering energy.

In this paper we will present simple formal proofs of some asymptotic approximations for the gamma function, like Stirling series and Gudermann series, and we will discuss several methods for approximately computing the phase shift, Eq. (1), from these asymptotic approximations in various regimes. We will compare these results with the corresponding ones obtained from other methods for computing phase shifts, like the WKB and the eikonal approximations. We will also present proofs of some exact relations for the phase shifts $\sigma_{l}$ which are more or less known in the literature. For instance, using Eq. (1) we will easily show that

$$
\sigma_{l}=\sigma_{0}+\sum_{m=1}^{l} \tan ^{-1}\left(\frac{\eta}{m}\right)
$$

and that

$$
\sigma_{0}=-\gamma \eta-\sum_{m=0}^{\infty}\left[\tan ^{-1}\left(\frac{\eta}{m+1}\right)-\frac{\eta}{m+1}\right]
$$

valid for all $\eta \in \mathbb{R}$, from which we obtain for $|\eta|<1$ the power series representation

$$
\sigma_{0}=-\gamma \eta-\sum_{k=1}^{\infty} \frac{(-1)^{k} \zeta(2 k+1)}{2 k+1} \eta^{2 k+1}
$$

where $\zeta$ is Riemann's zeta function and $\gamma$ is Euler's constant.

Using our asymptotic expansions we show that

$$
\sigma_{0}^{(1)}=\frac{1}{2} \tan ^{-1}(\eta)+\eta\left(\ln \left(\sqrt{1+\eta^{2}}\right)-1\right)-\frac{\eta}{12\left(1+\eta^{2}\right)}
$$

is an excellent approximation for the exact expression (3). We also show numerically that this approximation is very good even for small values of $\eta$. More generally, we show that

$$
\sigma_{l}^{(1)}=\left(l+\frac{1}{2}\right) \tan ^{-1}\left(\frac{\eta}{l+1}\right)+\eta\left(\ln \left(\sqrt{(l+1)^{2}+\eta^{2}}\right)-1\right)-\frac{\eta}{12\left((l+1)^{2}+\eta^{2}\right)}
$$

approximates (2) very well for $\sqrt{(l+1)^{2}+\eta^{2}}$ "large". 


\section{ASYMPTOTIC PROPERTIES OF THE GAMMA FUNCTION}

We start with the well-known integral representation for Euler's gamma function, valid for $\operatorname{Re}(z)>-1[5]$,

$$
\Gamma(1+z)=\int_{0}^{\infty} d t t^{z} e^{-t}
$$

For (1) we will take $z=l+i \eta$. Writing $t^{z}=e^{z \ln t}$, we can use the saddle point method to evaluate the integral

$$
\Gamma(1+z)=\int_{0}^{\infty} d t e^{-t+z \ln (t)} .
$$

Expanding the exponent of the integrand around its extremum, at $t_{0}=z$ and keeping terms up to second order,

$$
-t+z \ln (t) \simeq-z+z \ln (z)-\frac{1}{2 z}(t-z)^{2}
$$

Eq. (8) becomes

$$
\Gamma(1+z) \simeq e^{-z} z^{z} \int_{0}^{\infty} d t e^{-(t-z)^{2} / 2 z}
$$

For $\operatorname{Re}(z)=l \gg 1$ and $\operatorname{Im}(z)=\eta>0$, the Gaussian integral can be easily evaluated as

$$
\int_{0}^{\infty} d t e^{-(t-z)^{2} / 2 z}=\int_{-\infty}^{\infty} d t e^{-(t-z)^{2} / 2 z}=\sqrt{2 \pi z}
$$

and using this result in the previous equation, we obtain

$$
\Gamma(1+z) \simeq \sqrt{2 \pi} e^{-z} z^{z+1 / 2}
$$

This is the well-known Stirling's approximation for the gamma function, whose validity can be established for the whole complex plane, except the non-positive real axis, i.e., in $\mathbb{C}^{-}=\mathbb{C} \backslash(-\infty, 0]$, and for "large" values of $|z|$. See, e.g., Ref. [2].

On the real line, Stirling's approximation (12) has a long history having been first presented by de Moivre around 1730, who found an approximation for $n$ !, valid for "large" $n$, in the form $n ! \simeq K n^{n+\frac{1}{2}} e^{-n}$, for some unspecified constant $K$. In the same year, Stirling proved that $K=\sqrt{2 \pi}$ using Wallis product formula for $\pi$. This approximation became known as Stirling's approximation for $n$ ! and quite soon generalizations for Euler's gamma function on the positive real line became available. Stirling's approximation is very useful in Statistics and Probability Theory because if offers a very good approximation for "large" factorials. For small values of $n$, however, correcting factors are in order. Stirling found corrections for the approximation that bears his name for $n$ ! in terms of an asymptotic 
(but not convergent!) series in $1 / n$ that became known as Stirling's series. Further analysis of those corrections for Euler's gamma function $\Gamma(z)$, valid on the complex half-plane $\operatorname{Re}(z)>0$, have been performed by Binet in 1839 (Ref. [12]). Another important contribution was made by Gudermann in 1845 (Ref. [13]), who found another expression for the correcting factors in terms of a convergent expansion of another kind. The most important contribution to the study of corrections to Stirling's approximation on the complex plane was the work of Stieltjes, dated of 1889 (Ref. [14]), who generalized Stirling's approximation and Gudermann's corrections to the whole complex plane, excluding the negative real axis (i.e., to $\mathbb{C}^{-}$). For a more detailed account of the developments on the complex plane, see [2] and [11]. For some recent contributions to the correcting factors to factorials, see [15] and [16]. This last reference contains a list of historical results on corrections on Stirling's approximation.

Let us briefly describe the ideas behind Gudermann's corrections and present Stirling's series. Since Euler's gamma function satisfies $\Gamma(z+1)=z \Gamma(z)$, we get from $(12)$ the approximation

$$
\Gamma(z) \simeq \sqrt{2 \pi} e^{-z} z^{z-1 / 2}
$$

However, 13 contrasts with the expression obtained from (12) itself by replacing $z$ by $z-1$ :

$$
\Gamma(z) \simeq \sqrt{2 \pi} e^{-(z-1)}(z-1)^{z-1 / 2} .
$$

Since both expressions (13) and (14) are only valid for $|z|$ very large, there is no practical difference between them. Nevertheless, one can better deal with this situation by seeking an exact representation for $\Gamma$ in the whole region $\mathbb{C}^{-}$(and not only for "large" $\left.|z|\right)$ in the form

$$
\Gamma(z)=\sqrt{2 \pi} e^{-z} z^{z-1 / 2} e^{\mu(z)},
$$

and fixing the correction factor $e^{\mu(z)}$ by imposing the relation $\Gamma(z+1)=z \Gamma(z)$. A simple computation reveals that this condition implies that $\mu$ has to satisfy the functional equation

$$
\mu(z)-\mu(z+1)=\left(z+\frac{1}{2}\right) \ln \left(1+\frac{1}{z}\right)-1 .
$$

Moreover, the validity of (13) for "large" $|z|$ leads to the condition $\lim _{|z| \rightarrow \infty} \mu(z)=0$. This allows to a solution for (16). Indeed, it follows immediately from (16) that for any positive integer $n$ one has

$$
\mu(z)-\mu(z+n)=\sum_{m=0}^{n-1}\left[\left(z+m+\frac{1}{2}\right) \ln \left(1+\frac{1}{z+m}\right)-1\right]
$$


Hence, the condition $\lim _{|z| \rightarrow \infty} \mu(z)=0$ implies, in particular, that $\lim _{n \rightarrow \infty} \mu(z+n)=0$ and we get

$$
\mu(z)=\sum_{m=0}^{\infty}\left[\left(z+m+\frac{1}{2}\right) \ln \left(1+\frac{1}{z+m}\right)-1\right] .
$$

This series is known as Gudermann series. According to [2], it was first obtained by that author in 1845 [13] for real and positive $z$ and the generalization for $z \in \mathbb{C}^{-}$was obtained by Stieltjes in 1889 [14].

The series $(18)$ converges for all $z \in \mathbb{C}^{-}$and $\mu$ can be bounded by

$$
|\mu(z)| \leq \frac{1}{12} \frac{1}{\cos ^{2}(\varphi / 2)} \frac{1}{|z|},
$$

with $z=|z| e^{i \varphi}, z \in \mathbb{C}^{-}$(see [2] or [11]). On the real line, very precise upper and lower approximants of the $\Gamma$-function can be obtained from $(15)$ and $(18)$, valid also for small values of the argument (see [15] and [16]).

Notice that, by 19 , for $|z| \simeq 1$, the contribution of the factor $e^{\mu(z)}$ to 15 is lower than ci. $9 \%$ for $\varphi \simeq 0$ and lower than ci. $18 \% \varphi \simeq \pi / 2$. Hence, for the sake of precision, it is relevant in that range to consider corrections to Stirling's approximation (12).

With (15), and since $\Gamma(z+1)=z \Gamma(z)$, we can also write

$$
\Gamma(z+1)=\sqrt{2 \pi} e^{-z} z^{z+1 / 2} e^{\mu(z)} .
$$

Hence, $e^{\mu(z)}$ acts as a correcting factor for both $(12)$ and $(13)$. Beyond the Gudermann series (18), the function $\mu$ can be represented in many other forms. One of the most useful of them is the so-called Stirling series:

$$
\mu(z)=\sum_{n=1}^{\infty} \frac{B_{2 n}}{(2 n-1) 2 n} \frac{1}{z^{2 n-1}},
$$

where $B_{k}$ is the $k$-th Bernoulli number. One has to say that the series on the r.h.s. of (21) is an asymptotic series in $\mathbb{C}^{-}$, but is not convergent! See again [2]. Therefore, it is not to be seen as a Laurent expansion for $\mu$ around $z=0$. In fact, $z=0$ is not an isolated singularity of $\mu$, but a branch point, as we see from (18). Since (21) is an asymptotic series we can get good approximations for $\mu$ in $\mathbb{C}^{-}$by truncating it at some finite value of the index $n$ and taking $1 /|z|$ small enough. The first terms of 21 are

$$
\mu(z)=\frac{1}{12} \frac{1}{z}-\frac{1}{360} \frac{1}{z^{3}}+\frac{1}{1260} \frac{1}{z^{5}}+\cdots .
$$


For the correcting factor $e^{\mu(z)}$, this gives

$$
e^{\mu(z)}=1+\frac{1}{12} \frac{1}{z}+\frac{1}{288} \frac{1}{z^{2}}-\frac{139}{51840} \frac{1}{z^{3}}+\cdots .
$$

Therefore, we may write

$$
\begin{aligned}
\Gamma(z) & =\sqrt{2 \pi} e^{-z} z^{z-1 / 2}\left[1+\frac{1}{12} \frac{1}{z}+\frac{1}{288} \frac{1}{z^{2}}-\frac{139}{51840} \frac{1}{z^{3}}+\cdots\right], \\
\Gamma(z+1) & =\sqrt{2 \pi} e^{-z} z^{z+1 / 2}\left[1+\frac{1}{12} \frac{1}{z}+\frac{1}{288} \frac{1}{z^{2}}-\frac{139}{51840} \frac{1}{z^{3}}+\cdots\right] .
\end{aligned}
$$

These approximations are also known as Stirling's series for the gamma function. They are asymptotic (but not convergent!) expansions for $\Gamma$, valid for $z \in \mathbb{C}^{-}$and $1 /|z|$ "small". Although (24) are asymptotic but not convergent approximations for $\Gamma$, they can in some practical situations be more useful than the representation (15) or 20) with the convergent Gudermann expansion (18).

\section{STIRLING'S SERIES. A FORMAL DERIVATION}

The Stirling's series (24) leads to more accurate estimates of the phase-shifts (1) than Stirling's approximation (12), even when the value of $l$ is not "large". There are many proofs of Eqs. (21) or (24) in the real or in the complex domain (see, e.g., Ref. [2, 3]), but they are all rather involved. We will present now a simple derivation of Eq. (24b) (see also [4]).

Consider the curve $C$ in the complex $t$-plane parametrized by $s \in(-\infty, \infty)$ and satisfying the parabolic functional mapping [4, 6, 7],

$$
t(s)-z \ln (t(s))-A(z)=\frac{s^{2}}{2}
$$

with $A(z)=z-z \ln z$ and with $t(0)=z$. Defining $\omega(t)=t-z \ln (t)$, we can write

$$
\omega(t(s))=A(z)+\frac{s^{2}}{2} .
$$

One can choose $t(s)$ satisfying $t(s) \rightarrow 0$ as $t(s) \simeq e^{-\frac{s^{2}}{2 z}}$ for $s \rightarrow-\infty$ and $t(s) \simeq \frac{s^{2}}{2}+$ $i \operatorname{Im}(z) \ln \left(\frac{s^{2}}{2}\right)$ for $s \rightarrow+\infty$. By a continuous deformation of the $t$-integration curve in (7) from the positive real axis to $C$ and by carefully extending the integration to infinity, one can write, by Cauchy's theorem,

$$
\Gamma(1+z)=\int_{C} d t t^{z} e^{-t}=e^{-z+z \ln z} \int_{-\infty}^{\infty} e^{-s^{2} / 2} \frac{d t(s)}{d s} d s .
$$


The derivative $d t / d s$ can be written as a power series in $s^{2}$ using the mapping equation,

$$
\frac{d t}{d s}=\sum_{k=0}^{\infty} a_{2 k} s^{2 k}
$$

It is a simple matter to evaluate the coefficients $a_{k}$, by repeated differentiation of the mapping equation. We calculate below the first two terms, $a_{0}$, and $a_{2}$ (higher order terms can be derived similarly). Calling $\omega(t(s)) \equiv v(s)$, we write

$$
\begin{aligned}
\frac{d v(s)}{d s}= & \left(1-\frac{z}{t}\right) \frac{d t}{d s}=s \\
\frac{d^{2} v(s)}{d s^{2}}= & \left(1-\frac{z}{t}\right) \frac{d^{2} t}{d s^{2}}+\frac{z}{t^{2}}\left(\frac{d t}{d s}\right)^{2}=1 \\
\frac{d^{3} v(s)}{d s^{3}}= & \left(1-\frac{z}{t}\right) \frac{d^{3} t}{d s^{3}}+\frac{2 z}{t^{2}} \frac{d^{2} t}{d s^{2}} \frac{d t}{d s}-\frac{2 z}{t^{3}}\left(\frac{d t}{d s}\right)^{3}=0 \\
\frac{d^{4} v(s)}{d s^{4}}= & \left(1-\frac{z}{t}\right) \frac{d^{4} t}{d s^{4}}+\frac{4 z}{t^{2}} \frac{d^{3} t}{d s^{3}} \frac{d t}{d s}+\frac{3 z}{t^{2}}\left(\frac{d^{2} t}{d s^{2}}\right)^{2} \\
& -\frac{12 z}{t^{3}} \frac{d^{2} t}{d s^{2}}\left(\frac{d t}{d s}\right)^{2}+\frac{6 z}{t^{4}}\left(\frac{d t}{d s}\right)^{4}=0 .
\end{aligned}
$$

We now evaluate these equations at the extremum point, defined by the condition

$$
\left[\frac{d \omega(t)}{d t}\right]_{t_{0}}=0
$$

which is $t_{0}=z$ and $s=0$. The above four equations lead to the results

$$
\left[\frac{d t}{d s}\right]_{t=t_{0}}=\sqrt{z},\left[\frac{d^{3} t}{d s^{3}}\right]_{t=t_{0}}=\frac{1}{6 \sqrt{z}} \text { and }\left[\frac{d^{5}}{d s^{5}}\right]_{t=t_{0}}=\frac{1}{36 z^{3 / 2}}
$$

Thus the coefficients $a_{2 k}$ above are,

$$
\begin{gathered}
a_{0}=\left[\frac{d t}{d s}\right]_{t=t_{0}}=\sqrt{z} \\
a_{2}=\frac{1}{2 !}\left[\frac{d^{3} t}{d s^{2}}\right]_{t=t_{0}}=\frac{1}{12 \sqrt{z}}, \\
a_{4}=\frac{1}{4 !}\left[\frac{d^{5} t}{d s^{5}}\right]_{t=t_{0}}=\frac{1}{4 !} \frac{1}{36 z^{3 / 2}} .
\end{gathered}
$$

Using the Gaussian integral formula,

$$
\int_{-\infty}^{\infty} d s e^{-s^{2} / 2} s^{n} d s=\sqrt{2 \pi}(n-1) ! !
$$


for $n$ even, the full integral in the $\Gamma$ function can be written down in the form of the Stirling series above, namely,

$$
\Gamma(z+1)=\sqrt{2 \pi} e^{-z} z^{z} \sum_{k=0}^{\infty}(2 k-1) ! ! a_{2 k} .
$$

Thus, evaluating the coefficients $a_{2 k}$ and inserting above, we get

$$
\Gamma(z+1)=\sqrt{2 \pi} e^{-z} z^{z+1 / 2}\left[1+\frac{1}{12 z}+\frac{1}{288 z^{2}}+\cdots\right] .
$$

The use of the quadratic mapping analysis of integrals and the generation of appropriate asymptotic series in general was demonstrated in, e.g., [7] for the case of the Gamow integral employed in nuclear astrophysics. This integral has the general form,

$$
I(a)=\int_{0}^{\infty} d x S(x) \exp \left(-x-\frac{a}{x^{1 / 2}}\right)
$$

where the function $S(x)$ is usually a slowly varying function of $x$ and can be written as a sum $S(x)=\sum_{k=0}^{\infty} S_{k} x^{k}$, which would then result in a series representation of the Gamow integral similar to what was done for the $\Gamma$ function above.

We now use the above results to evaluate the Coulomb phase-shifts (1).

\section{AN EXACT EXPRESSION FOR THE PHASE SHIFT AND FIRST APPROXIMATIONS}

From (1) and (15), and using the identity

$$
\ln \left(\frac{x+i y}{x-i y}\right)=2 i \tan ^{-1}\left(\frac{y}{x}\right)
$$

valid for $x, y \in \mathbb{R}$ with $x>0$, we get after some elementary computations the following exact expression for the phase shift:

$$
\sigma_{l}=\sigma_{l}^{(0)}+M_{l, \eta}
$$

where we define

$$
\sigma_{l}^{(0)} \equiv\left(l+\frac{1}{2}\right) \tan ^{-1}\left(\frac{\eta}{l+1}\right)+\eta\left(\ln \left(\sqrt{(l+1)^{2}+\eta^{2}}\right)-1\right)
$$

and $M_{l, \eta} \equiv \frac{1}{2 i}(\mu(1+l+i \eta)-\mu(1+l-i \eta))$. Below, we will use the series expansion (18) to find closed expressions for $M_{l, \eta}$, for $\sigma_{l}$ and, in particular, for $\sigma_{0}$. Before we proceed let us make some comments about some useful approximation we can obtain from (36)-(37). 
Using $(19)$ with $|z|=\sqrt{(l+1)^{2}+\eta^{2}}$ and $\varphi=\tan ^{-1}\left(\frac{\eta}{l+1}\right)$, one finds the bound

$$
\left|M_{l, \eta}\right| \leq \frac{1}{6\left(l+1+\sqrt{(l+1)^{2}+\eta^{2}}\right)} .
$$

Hence, for $l \gg 1$ or $|\eta| \gg 1$ the contribution of $M_{l, \eta}$ to (36) can be neglected and we can restrict as a first approximation to $\sigma_{l}^{(0)}$.

For $l=0$, for instance, one gets from (37) the approximation

$$
\sigma_{0} \simeq \sigma_{0}^{(0)}=\frac{1}{2} \tan ^{-1}(\eta)+\eta\left(\ln \left(\sqrt{1+\eta^{2}}\right)-1\right)
$$

with an error bounded by $\frac{1}{6}\left(1+\sqrt{1+\eta^{2}}\right)^{-1}$. For $|\eta| \gg 1$, Eq. 38 gives the approximation

$$
\sigma_{0} \simeq \frac{\pi}{4}+\eta(\ln (\eta)-1)
$$

For $\frac{|\eta|}{l+1} \ll 1$ and $l \gg 1$ we get from 37 the approximation

$$
\sigma_{l} \simeq \eta \ln (l+1)
$$

\section{CLOSED EXPRESSION FOR THE PHASE SHIFT}

Now we will try to find closed expressions for $\sigma_{l}$ and $\sigma_{0}$. According to (1), using the fact that $\Gamma(z+1)=z \Gamma(z)$ for all $z \in \mathbb{C}^{-}$, we have

$$
e^{2 i \sigma_{l}}=\frac{\Gamma(1+l+i \eta)}{\Gamma(1+l-i \eta)}=\frac{(l+i \eta) \cdots(1+i \eta)}{(l-i \eta) \cdots(1-i \eta)} \frac{\Gamma(1+i \eta)}{\Gamma(1-i \eta)}=\frac{(l+i \eta) \cdots(1+i \eta)}{(l-i \eta) \cdots(1-i \eta)} e^{2 i \sigma_{0}} .
$$

Therefore $\sigma_{l}=\sigma_{0}+\frac{1}{2 i} \sum_{m=1}^{l} \ln \left(\frac{m+i \eta}{m-i \eta}\right)$ and using 35 , one has

$$
\sigma_{l}=\sigma_{0}+\sum_{m=1}^{l} \tan ^{-1}\left(\frac{\eta}{m}\right)
$$

Eq. (41) is a remarkable expression, since it shows that $\sigma_{l}$ differs from $\sigma_{0}$ by a finite sum. Let us now find a more explicit expression for $\sigma_{0}$. According to (36)- 37),

$$
\sigma_{0}=\frac{1}{2} \tan ^{-1}(\eta)+\eta\left(\ln \left(\sqrt{1+\eta^{2}}\right)-1\right)+M_{0, \eta}
$$

Now we analyze $M_{0, \eta} \equiv \frac{1}{2 i}(\mu(1+i \eta)-\mu(1-i \eta))$ more closely. According to 18 (with the change of summation variable $m \rightarrow m-1$ ), we have,

$$
M_{0, \eta}=\frac{1}{2 i} \sum_{m=1}^{\infty}\left[\left(m+i \eta+\frac{1}{2}\right) \ln \left(1+\frac{1}{m+i \eta}\right)-\left(m-i \eta+\frac{1}{2}\right) \ln \left(1+\frac{1}{m-i \eta}\right)\right] \text {. }
$$


After simple rearrangements and using we can write 443 as

$$
M_{0, \eta}=\frac{1}{2 i} \sum_{m=1}^{\infty}\left[\left(m+\frac{1}{2}\right)\left(\ln \left(\frac{m+1+i \eta}{m+1-i \eta}\right)-\ln \left(\frac{m+i \eta}{m-i \eta}\right)\right)+i \eta\left(\ln \left((m+1)^{2}+\eta^{2}\right)-\ln \left(m^{2}+\eta^{2}\right)\right.\right.
$$

Using (35) and defining

$$
A_{m} \equiv \frac{1}{2 i} \ln \left(\frac{m+i \eta}{m-i \eta}\right)=\tan ^{-1}\left(\frac{\eta}{m}\right) \quad \text { and } \quad B_{m} \equiv \frac{\eta}{2} \ln \left(m^{2}+\eta^{2}\right)
$$

we can write 44 as $M_{0, \eta}=\lim _{N \rightarrow \infty} M_{0, \eta}^{N}$, where

$$
M_{0, \eta}^{N}=\sum_{m=1}^{N}\left[\left(m+\frac{1}{2}\right)\left(A_{m+1}-A_{m}\right)+B_{m+1}-B_{m}\right] .
$$

Now,

$$
\sum_{m=1}^{N}\left(B_{m+1}-B_{m}\right)=B_{N+1}-B_{1}
$$

and

$$
\begin{aligned}
\sum_{m=1}^{N}\left(m+\frac{1}{2}\right)\left(A_{m+1}-A_{m}\right) & =\sum_{m=1}^{N}\left[\left((m+1)+\frac{1}{2}\right) A_{m+1}-\left(m+\frac{1}{2}\right) A_{m}\right]-\sum_{m=1}^{N} A_{m+1} \\
& =\left(N+1+\frac{1}{2}\right) A_{N+1}-\left(1+\frac{1}{2}\right) A_{1}-\sum_{m=1}^{N} A_{m+1}
\end{aligned}
$$

Hence, collecting the results, we have

$$
\begin{aligned}
M_{0, \eta}= & \lim _{N \rightarrow \infty}\left[\left(N+\frac{3}{2}\right) \tan ^{-1}\left(\frac{\eta}{N+1}\right)+\frac{\eta}{2} \ln \left((N+1)^{2}+\eta^{2}\right)-\sum_{m=1}^{N} \tan ^{-1}\left(\frac{\eta}{m+1}\right)\right] \\
& -\frac{3}{2} \tan ^{-1}(\eta)-\frac{\eta}{2} \ln \left(1+\eta^{2}\right) \\
= & \eta-\frac{3}{2} \tan ^{-1}(\eta)-\frac{\eta}{2} \ln \left(1+\eta^{2}\right)+\lim _{N \rightarrow \infty}\left[\eta \ln (N+1)-\sum_{m=1}^{N} \tan ^{-1}\left(\frac{\eta}{m+1}\right)\right] .
\end{aligned}
$$

Now, adding and subtracting $\eta \sum_{m=0}^{N} \frac{1}{m+1}$ to the terms in brackets, whose limit is being taken in 48), we get

$$
\eta \ln (N+1)-\sum_{m=1}^{N} \tan ^{-1}\left(\frac{\eta}{m+1}\right)=\eta\left[\ln (N+1)-\sum_{m=0}^{N} \frac{1}{m+1}\right]-\sum_{m=0}^{N}\left[\tan ^{-1}\left(\frac{\eta}{m+1}\right)-\frac{\eta}{m+1}\right]+\tan ^{-}
$$

With this, 48 becomes

$$
M_{0, \eta}=\eta(1-\gamma)-\frac{1}{2} \tan ^{-1}(\eta)-\frac{\eta}{2} \ln \left(1+\eta^{2}\right)-\sum_{m=0}^{\infty}\left[\tan ^{-1}\left(\frac{\eta}{m+1}\right)-\frac{\eta}{m+1}\right],
$$




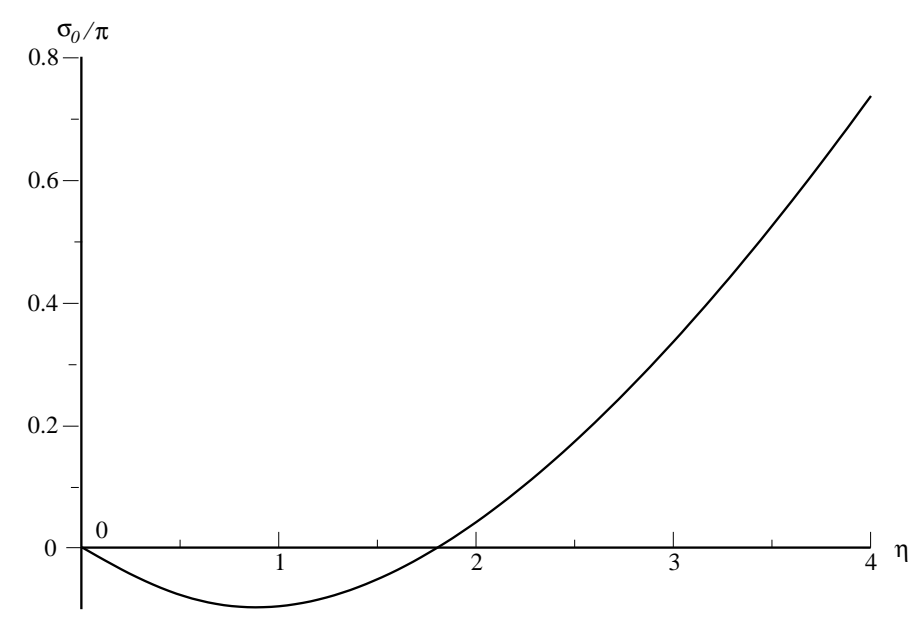

FIG. 1: The graph of $\sigma_{0} / \pi$ according to the exact formula (50) for $0 \leq \eta \leq 4$. Notice that $\sigma_{0}$ vanishes at $\eta \simeq 1.810$.

where $\gamma \equiv \lim _{N \rightarrow \infty}\left[\sum_{m=0}^{N} \frac{1}{m+1}-\ln (N+1)\right]$ is Euler's constant $\gamma \simeq 0.5772156649 \ldots$.

Inserting 49) into (42) we finally get, after some trivial cancelations,

$$
\sigma_{0}=-\gamma \eta-\sum_{m=0}^{\infty}\left[\tan ^{-1}\left(\frac{\eta}{m+1}\right)-\frac{\eta}{m+1}\right]
$$

By recalling the Taylor expansion of $\tan ^{-1}$ about 0 ,

$$
\tan ^{-1}(x)=\sum_{k=0}^{\infty} \frac{(-1)^{k}}{2 k+1} x^{2 k+1}
$$

we can write (50) for $|\eta|<1$ as

$$
\sigma_{0}=-\gamma \eta-\sum_{m=0}^{\infty} \sum_{k=1}^{\infty} \frac{(-1)^{k}}{2 k+1}\left(\frac{\eta}{m+1}\right)^{2 k+1}=-\gamma \eta-\sum_{k=1}^{\infty} \frac{(-1)^{k} \zeta(2 k+1)}{2 k+1} \eta^{2 k+1},
$$

where $\zeta$ is Riemann's zeta function.

In Figure 1 we plot $\sigma_{0}$ for $0 \leq \eta \leq 4$. As we discuss below, for larger values $\sigma_{0}$ follows very closely the behavior dictated by the asymptotic approximations (38) or (39). In Figure 1 we see that $\sigma_{0}$ vanishes at $\eta=0$ and $\eta \simeq 1.810$. This latter value of $\eta$ is coincidentally quite close to the value of $\sqrt{2}$, which was obtained in [17] in connection with the scattering of identical charged particles (Fermions or Bosons). The Mott cross section, at this critical value of $\eta=\sqrt{2}$, was predicted to be isotropic over a broad range of angles around $90^{\circ}$. 


\section{THE PHASE SHIFT AND STIRLING'S SERIES}

The above asymptotic formulae (37), (38), 39) and (40) will be discussed further below within the low-energy, large- $\eta$, large- $l$ case of the WKB approximation, and the high-energy, small- $\eta$, large- $l$ case of the eikonal approximation. In this section we use Stirling series for the gamma function (24) to further improve those approximations.

A first order correction to Eq. (37) can be easily evaluated using Stirling's series (22)-24). For this purpose, we rewrite Eq. (1) in the form

$$
e^{2 i \sigma_{l}}=e^{2 i \sigma_{l}^{(0)}} F(z)
$$

with $z=1+l+i \eta$ and $\sigma_{l}^{(0)}$ given in 37 , where

$$
F(z)=e^{\mu(z)-\mu\left(z^{*}\right)}=\exp \left(\frac{1}{12}\left(\frac{1}{z}-\frac{1}{z^{*}}\right)-\frac{1}{360}\left(\frac{1}{(z)^{3}}-\frac{1}{\left(z^{*}\right)^{3}}\right)+\cdots\right) .
$$

where the $*$ symbol refers to complex conjugation. The first order approximation for $F(z)$ is

$$
F^{(1)}(z) \equiv \exp \left(\frac{1}{12}\left(\frac{1}{z}-\frac{1}{z^{*}}\right)\right)=: e^{2 i \Delta \sigma_{l}^{(0)}} .
$$

Evaluating the above expression, we find

$$
\Delta \sigma_{l}^{(0)}=-\frac{\eta}{12\left((l+1)^{2}+\eta^{2}\right)} .
$$

Therefore, the first order approximation to the Coulomb phase-shifts is

$$
\begin{aligned}
\sigma_{l}^{(1)} & =\sigma_{l}^{(0)}+\Delta \sigma_{l}^{(0)} \\
& =\left(l+\frac{1}{2}\right) \tan ^{-1}\left(\frac{\eta}{l+1}\right)+\eta\left(\ln \left(\sqrt{(l+1)^{2}+\eta^{2}}\right)-1\right)-\frac{\eta}{12\left((l+1)^{2}+\eta^{2}\right)}
\end{aligned}
$$

Figure 2 shows Coulomb phase-shifts versus $l$.

From 56 we get, in particular, the first order correction to 38 due to Stirling's series:

$$
\begin{aligned}
\sigma_{0}^{(1)} & =\sigma_{0}^{(0)}+\Delta \sigma_{0}^{(0)} \\
& =\frac{1}{2} \tan ^{-1}(\eta)+\eta\left(\ln \left(\sqrt{1+\eta^{2}}\right)-1\right)-\frac{\eta}{12\left(1+\eta^{2}\right)}
\end{aligned}
$$

It is interesting to compare the approximation (57) to our exact expression for $\sigma_{0}$ given in 3. Figure 3 we plot the relative error $\frac{\sigma_{0}^{(1)}-\sigma_{0}}{\sigma_{0}}$ for values of the Sommerfeld parameter between 0 and 5 . It shows that $\sigma_{0}^{(1)}$ is an excellent approximation for $\sigma_{0}$, with relative errors below $1 \%$, even for "small" values of $\eta$, except, perhaps, near $\eta \simeq 1.81$, where $\sigma_{0}$ vanishes. 


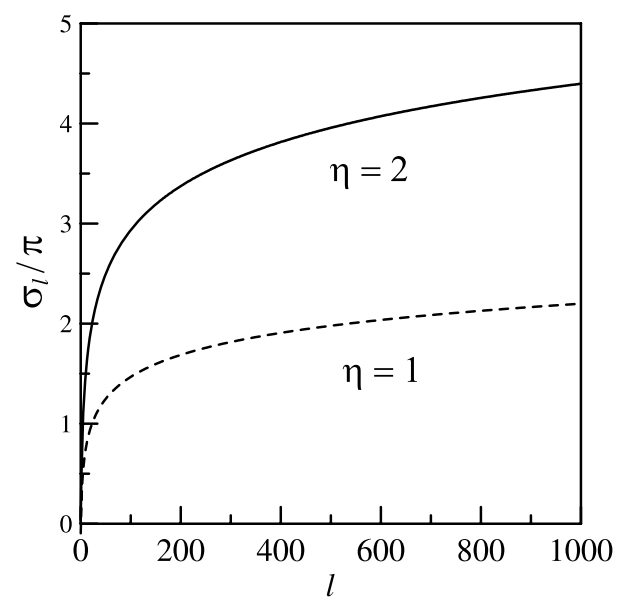

FIG. 2: Coulomb phase-shifts as functions of $l$. Results normalized with respect to $\pi$ are given for two values of the Sommerfeld parameter.

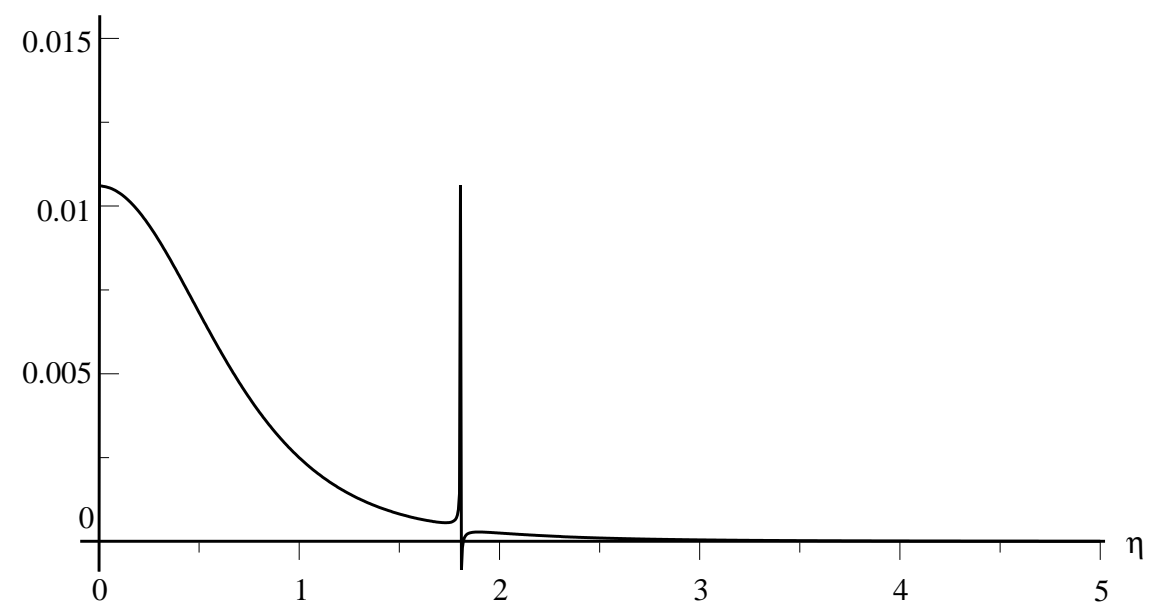

FIG. 3: The relative error $\frac{\sigma_{0}^{(1)}-\sigma_{0}}{\sigma_{0}}$ for $0 \leq \eta \leq 5$. The sharp peak around $\eta \simeq 1.81$ is due the the vanishing of $\sigma_{0}$ at that point.

\section{DISCUSSION OF THE RESULTS FOR STIRLING'S SERIES}

The lowest orders approximations (in 1/z) of the previous section are supposed to work for $|z| \gg 1$, which means $l \gg 1$ and/or $\eta \gg 1$. They are, in fact, very accurate, even when these conditions are not well satisfied. A first illustration of this fact is presented in Figure 4. where we compare the exact phase-shifts (solid line) with the lowest order approximation $\sigma_{l}^{(0)}$ (open circles) for a small $\eta$, as functions of $l$. They are very close. The only exception 


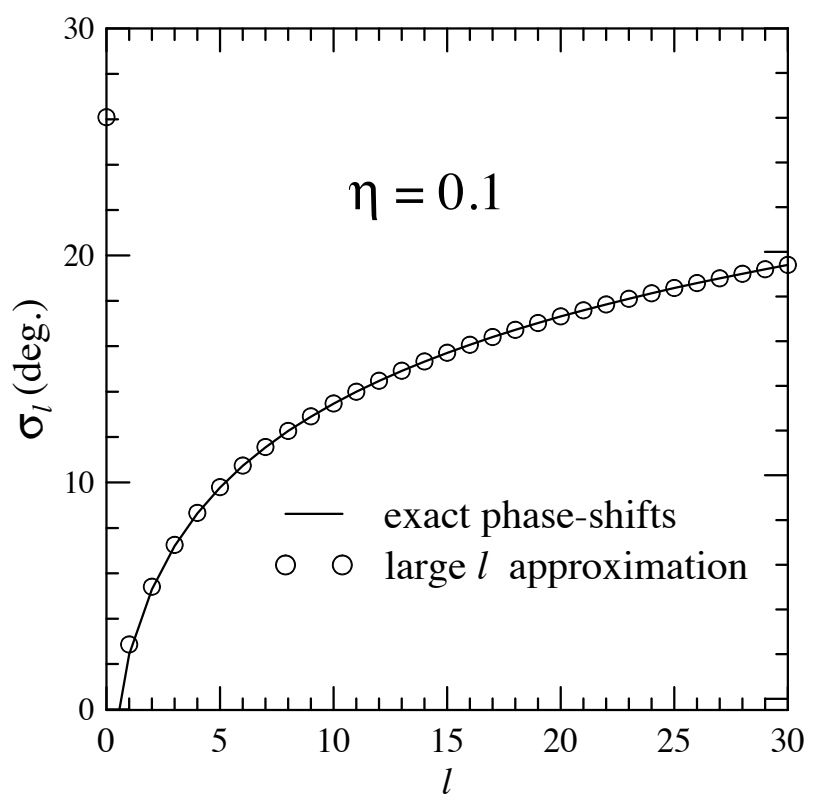

FIG. 4: The approximate Coulomb phase-shifts $\sigma_{l}^{(0)}$ and $\sigma_{l}^{(1)}$ for $\eta=0.1$, as functions of $l$. For details see the text.

is the case of $l=0$, where they are quite different.

For larger values of $\eta$, the agreement is much better. This can be seen in Table $\mathbb{I}$, where we compare the two approximations of the previous section with the exact Coulomb phaseshifts. Note that the $\sigma_{l}^{(1)}$ is very accurate even for $l=0$ and $\eta=0.1$.

This point can be seen more clearly in Figure 5, where the exact s-wave phase-shifts are compared with the approximations $\sigma_{l=0}^{(0)}$ and $\sigma_{l=0}^{(1)}$. The comparison indicates that the usual large- $\eta$ approximation, $\sigma_{l=0}^{(0)}$, is very poor. The situation is completely different with the improved approximation, $\sigma_{l=0}^{(1)}$, which includes the first order correction of Stirling's series. In this case, one gets accurate results for any value of the Sommerfeld parameter, $\eta$.

\section{COMPARISON WITH OTHER APPROXIMATIONS}

We turn now to well known approximations used to calculate the phase shifts, motivated by the physical conditions. The first such approximation is the WKB one invoked to consider scattering under semi-classical conditions of short local wave lengths. In this approximation, 


$$
\eta \quad l \quad \sigma_{l}^{(0)} / \pi \quad \sigma_{l}^{(1)} / \pi \quad \sigma_{l}^{\text {exact }} / \pi
$$

$\begin{array}{lllll}0.1 & 0 & -0.01581 & -0.01844 & -0.01825 \\ & 1 & 0.01413 & 0.01346 & 0.01348 \\ & 2 & 0.02967 & 0.02930 & 0.02938\end{array}$

\begin{tabular}{ccccc}
1.0 & 0 & -0.08299 & -0.09625 & -0.09602 \\
& 1 & 0.1592 & 0.1539 & 0.1540 \\
& 2 & 0.3042 & 0.3015 & 0.3016 \\
\hline
\end{tabular}

TABLE I: Coulomb phase-shifts as functions of $l$, for two values of the Sommerfeld parameter.

one finds for the phase shift the following expression [1],

$$
\sigma_{l}=\lim _{r \rightarrow \infty}\left[\int_{r_{l}}^{r} d r^{\prime} k_{l}\left(r^{\prime}\right)-\int_{r_{l}^{(0)}}^{r} d r^{\prime} k_{l}^{(0)}\left(r^{\prime}\right)\right]
$$

where $k_{l}(r)$ and $k_{l}^{(0)}(r)$ are the local wave numbers in the presence and absence of the potential, respectively,

$$
\begin{gathered}
\hbar k_{l}(r)=p_{l}(r)=\sqrt{2 \mu\left(E-V(r)-\frac{\hbar^{2}(l+1 / 2)^{2}}{2 \mu r^{2}}\right)}, \\
\hbar k_{l}^{(0)}(r)=p_{l}^{(0)}(r)=\sqrt{2 \mu\left(E-\frac{\hbar^{2}(l+1 / 2)^{2}}{2 \mu r^{2}}\right)},
\end{gathered}
$$

where $\mu$, here, is the reduced mass. The radii, $r_{l}$ and $r_{l}^{(0)}$, are the classical turning points defined by $p_{l}\left(r_{l}\right)=0$, and $p_{l}^{(0)}\left(r_{l}^{(0)}\right)=0$, respectively. The phase shift, now a function of the energy (or the asymptotic wave number $k$ ), and the semi-classical angular momentum, $\lambda=l+1 / 2$, can be evaluated once the potential is given. In the case of point-charge Coulomb scattering, the result of such a calculation results in the following expression for the WKB phase shift function $\delta(\lambda, k)=\sigma(\lambda, k)$,

$$
\sigma(\lambda, k)=\frac{1}{2} \eta \ln \left[\eta^{2}+\lambda^{2}\right]+\lambda \sin ^{-1}\left[\frac{\eta}{\sqrt{\eta^{2}+\lambda^{2}}}\right]-\eta
$$




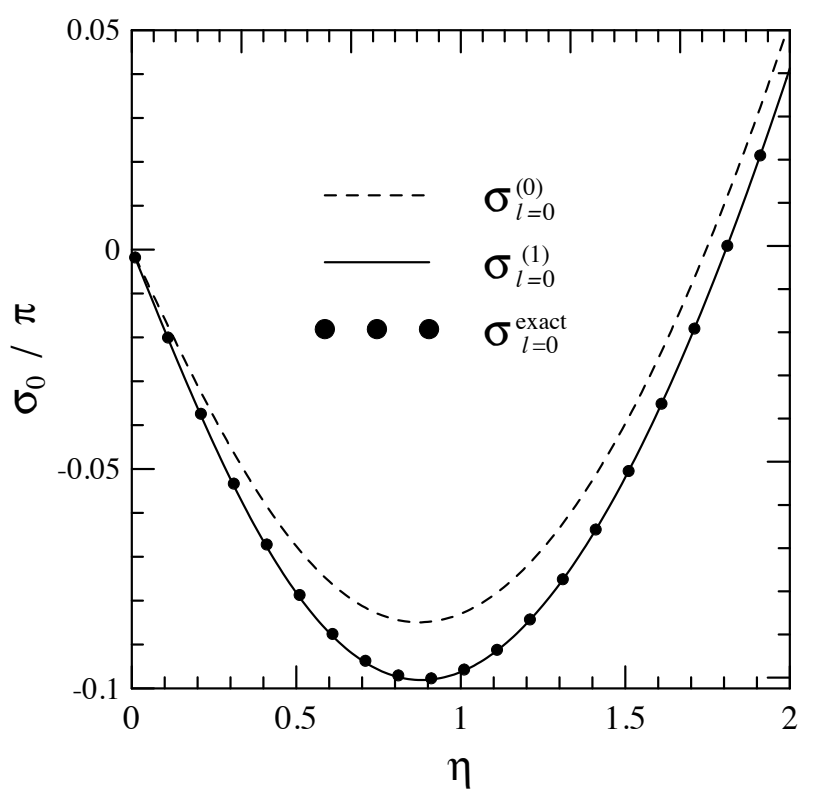

FIG. 5: Coulomb s-wave phase-shifts as a function of the Sommerfeld parameter. Exact values are compared with the approximations discussed in the text.

where the Sommerfeld parameter $\eta$ is related to the asymptotic wave number, $k$, by, $\eta=k a$, where $a$ is half the distance of closest approach for head-on, zero impact parameter, collision.

The above expression should be compared to our Eq. (37). Clearly the major difference resides in the introduction of the semi-classical angular momentum variable $\lambda$ in the former equation Eq. (61). This change is in fact necessary when the WKB form of the wave function is invoked [8], to guarantee the presence of a classical turning point for s-waves (now the angular momentum is not zero but rather $\hbar / 2$ ).

We turn next to the eikonal approximation. This, may be considered as the high-energy limit of the WKB approximation. Expanding the WKB phase shift in V/E, and keeping the linear term, and further assuming $r^{2}=z^{2}+b^{2}$, with $b$ being the impact parameter, $k b=\lambda$, we find for the eikonal Coulomb phase shift, $\sigma(b, k)$, the following,

$$
\sigma(b, k)=-\frac{\eta}{2} \int_{-\infty}^{\infty} d z \frac{1}{\sqrt{b^{2}+z^{2}}}
$$

The integral in the above equation diverges at both extremes. Ref. [9] introduced a screening function that renders the integral finite. The screening function considered is 
$F(r)=\Theta(a-r)$, where the screening length $a$ is of very large, and taken to be $a \gg b$. Then,

$$
\sigma(b, k)=-\frac{\eta}{2} \ln \left(\frac{2 a+2 \sqrt{a^{2}-b^{2}}}{2 a-2 \sqrt{a^{2}-b^{2}}}\right),
$$

which, with $a \gg b$, reduces to

$$
\sigma(b, k)=\eta \ln \left(\frac{b}{2 a}\right)=\eta \ln \lambda-\eta \ln (2 k a) .
$$

The above form is similar to Eq. (40), except for the change $l \rightarrow \lambda$ and the screening term, which depends only on energy and not on $\lambda$. Glauber [9] considered also and exponential screening function of the form $F(r)=\exp -r / a$, and found the following limiting expression for the Coulomb phase,

$$
\sigma(b, k)=\eta\left[\ln \frac{b}{2 a}-\gamma\right],
$$

where $\gamma$ is Euler's constant, $\gamma=0.57721 \ldots$ Again, the main feature of Eq. 40 of a logarithmic dependence on $b$, and thus $\lambda$ is maintained. A Gaussian shaped screening function $F(r)=\exp \left[-r^{2} / a^{2}\right]$ was considered by [10], and the result found for the phase shift is,

$$
\sigma(b, k)=\eta\left[\ln \frac{b}{2 a}-\frac{\gamma}{2}\right] .
$$

Once again the main feature of Eq. (40) is maintained, namely, the logarithmic dependence on b. Clearly the discussion above demonstrates that the best route to follow to obtain well behaved and defined asymptotic forms for the Coulomb phase shift is to rely on the exact expression, Eq. (1), and use the uniform approximation method of Stirling's series to obtain the correct asymptotic form of the $\Gamma$ function.

\section{QUANTUM AND CLASSICAL COULOMB DEFLECTION FUNCTIONS}

An important theoretical entity which enters in any semi-classical treatment of scattering is the deflection function. In the continuous $\lambda=l+1 / 2$ limit, the deflection function is just the derivative of $2 \sigma(\lambda, k)$ with respect to $\lambda[1]$. Thus,

$$
\Theta(\lambda)=2 \frac{d \sigma(\lambda, k)}{d \lambda}
$$


Using the WKB expression, Eq. (61), we obtain the well known classical Rutherford deflection function,

$$
\Theta(\lambda)=2 \tan ^{-1} \frac{\eta}{\lambda}
$$

The above should be compared to the exact "quantum" deflection function obtained from $\sigma_{l}$ of Eq. (2), which can be re-written as a recursion formula, viz,

$$
\sigma_{l}=\sigma_{l-1}+\tan ^{-1} \frac{\eta}{l}
$$

Clearly one can define the derivative as merely the difference, $\sigma_{l}-\sigma_{l-1}$, and thus the quantum deflection function, $\Theta_{q}(l)$ is,

$$
\Theta_{q}(l)=2\left[\sigma_{l}-\sigma_{l-1}\right]=2 \tan ^{-1} \frac{\eta}{l} .
$$

The quantum and classical deflection functions agree if one makes the change $l \rightarrow \lambda$, as expected.

\section{CONCLUSIONS}

In this paper we have revisited the literature on the point Coulomb phase shift, and sharpened the applicability and range of validity of several of the approximations usually employed to calculate it . In particular, the phase shift at zero angular momentum, $\sigma_{0}(\eta)$, as a function of the Sommerfeld parameter, $\eta$, is calculated exactly and a simple analytic expression for it valid for small and large $\eta$ is found. This is useful when evaluating the phase shift as a function of angular momentum, $l$, using the exact formula, $\sigma_{l}(\eta)=\sigma_{0}(\eta)+\sum_{m=0}^{l} \tan ^{-1}(\eta / m)$, of Eq. (2).

\section{Acknowledgments}

This work was supported in part by the CNPq, FAPESP and the MCT-National Institute of Quantum Information.

[1] See, e.g., D. M. Brink, Semi-classical methods for nucleus-nucleus scattering, Cambridge University Press (London \& New York, 1985). 
[2] Reinhold Remmert. Classical Topics in Complex Function Theory. Graduate Texts in Mathematics. Springer Verlag, New York (1998).

[3] G. H. Hardy. Divergent Series. Second Edition (textually unaltered). AMS Chelsea Publishing. American Mathematical Society, Providence, Rhode Island (1991).

[4] Harry Hochstadt. The Functions of Mathematical Physics. Dover Publications Inc. (New York, 1971).

[5] M. Abramowitz and I. A. Stegun, Handbook of Mathematical Functions, Dover Publ. (New York, 1970).

[6] R. B. Dingle, Asymptotic Expansions: their derivation and interpretation Academic Press (New York \& London, 1973).

[7] M. S. Hussein and M. P. Pato, Brazilian Journal of Physics, 27, 364 (1997).

[8] R. E. Langer, Phys. Rev. 51, 545 (1937).

[9] R. J. Glauber, Lectures in Theoretical Physics vol. I, ed. W. E. Brittin (Boulder: University of Colorado Press) 315-414 (1959).

[10] M. A. Hassan, J. Phys. G: Nucl. Phys. 12, 1233 (1986)

[11] Reinhold Remmert, Wielandt's Theorem About the $\Gamma$-function, Amer. Math. Monthly, 103, N0. 3, 214-220 (1996).

[12] M. J. Binet, "Mémoire sur les intégrales définies Eulériennes", Journ. de l'École Roy. Polyt. 16, 123-343 (1839).

[13] C. Gudermann. Journ. reine angew. Math. 29, 209-212 (1845).

[14] T.-J. Stieltjes, "Sur le developement de $\log \Gamma(a)$ ". Journ. Math. Pur. Appl. (4)5, 425-444 (1889).

[15] H. Robbins, "A Remark on Stirling's Formula", Amer. Math. Monthly, 62, 26-29 (1955).

[16] M. Mansour "Note on Stirling's Formula", International Mathematical Forum 4, no. 31, 1529$1534(2009)$.

[17] L. F. Canto, R. Donangelo, and M. S. Hussein, Modern Physics Letters A 16, 1027 (2001) 